This is the author's final, peer-reviewed manuscript as accepted for publication. The publisher-formatted version may be available through the publisher's web site or your institution's library.

\title{
Naïve rat umbilical cord matrix stem cells significantly attenuate mammary tumor growth through modulation of endogenous immune responses
}

Atsushi Kawabata, Naomi Ohta, Garret Seiler, Marla M. Pyle, Susumu Ishiguro, Yongqing Zhang, Kevin G. Becker, Deryl Troyer, and Masaaki Tamura

\section{How to cite this manuscript}

If you make reference to this version of the manuscript, use the following information:

Kawabata, A., Ohta, N., Seiler, G., Pyle, M. M., Ishiguro, S., Zhang, Y., ...Tamura, M. (2013). Naïve rat umbilical cord matrix stem cells significantly attenuate mammary tumor growth through modulation of endogenous immune responses. Retrieved from http://krex.ksu.edu

\section{Published Version Information}

Citation: Kawabata, A., Ohta, N., Seiler, G., Pyle, M. M., Ishiguro, S., Zhang, Y. Q., ...Tamura, M. (2013). Naïve rat umbilical cord matrix stem cells significantly attenuate mammary tumor growth through modulation of endogenous immune responses. Cytotherapy, 15(5), 586-597.

Copyright: Copyright (c) 2013, International Society for Cellular Therapy.

Digital Object Identifier (DOI): doi:10.1016/j.jcyt.2013.01.006

Publisher's Link: http://www.sciencedirect.com/science/article/pii/S1465324913001199

This item was retrieved from the K-State Research Exchange (K-REx), the institutional repository of Kansas State University. K-REx is available at http://krex.ksu.edu 


\title{
Naïve rat umbilical cord matrix stem cells significantly attenuate mammary tumor growth through modulation of endogenous immune responses
}

\author{
Atsushi Kawabata ${ }^{1}$, Naomi Ohta $^{1}$, Garret Seiler ${ }^{1}$, Marla M. Pyle ${ }^{1}$, Susumu Ishiguro ${ }^{1}$, Yongqing \\ Zhang $^{2}$, Kevin G. Becker ${ }^{2}$, Deryl Troyer ${ }^{1}$, and Masaaki Tamura ${ }^{1}$ * \\ ${ }^{1}$ Department of Anatomy \& Physiology, Kansas State University, College of Veterinary \\ Medicine, Manhattan, KS 66506; ${ }^{2}$ Gene Expression and Genomics Unit, NIH Biomedical \\ Research Center, National Institute on Aging, NIH, Baltimore, MD 21224
}

Running Title: Rat UCMSC enhance immune response to breast cancer

Key words: rat umbilical cord matrix stem cells, mammary tumor, immune response, $\mathrm{T}$ cells, macrophages

Novelty: This is the first study demonstrating mechanisms by which naive rUCMSC cytotherapy completely regress orthotopic mammary carcinoma grafts. The rUCMSC stimulated infiltration of CD8+ and CD4+ T cells and NK cells and decreased CD68+ monocytes/macrophages and Foxp3+ regulatory $\mathrm{T}$ cells in tumor tissues. Microarray analysis and Boyden chamber migration assays identified that MCP-1 is crucial for this lymphocyte infiltration. UCMSC-based cytotherapy can be a new therapeutic modality for the treatment of mammary carcinoma. 
* Correspondence to:

Masaaki Tamura, Associate Professor

Department of Anatomy \& Physiology,

Kansas State University, College of Veterinary Medicine

Manhattan, KS 66506

Phone: (785) 532-4825, Fax: (785) 532-4557

Email: mtamura@,vet.ksu.edu 


\section{Abstract}

Background- Un-engineered human and rat umbilical cord matrix stem cells (rUCMSC)

attenuate growth of several types of tumors in mice and rats. However, the mechanism by which UCMSC attenuate tumor growth has not been studied rigorously.

Methods- The possible mechanisms of tumor growth attenuation by rUCMSC were studied using orthotopic Mat B III rat mammary tumor grafts in female F344 rats. Tumor-infiltrating leukocytes were identified and quantified by immunohistochemical image analysis. Potential cytokines involved in lymphocyte infiltration in the tumors were determined by microarray and Western blot analysis. The Boyden chamber migration assay was performed for the functional analysis of identified cytokines.

Results- rUCMSC markedly attenuated the tumor growth; this attenuation was accompanied by considerable lymphocyte infiltration. Immunohistochemical analysis revealed that the majority of infiltrating lymphocytes in the rUCMSC-treated tumors were CD3+ T cells. In addition, treatment with rUCMSC significantly increased infiltration of CD $8+$ and CD4+ T cells and NK cells throughout tumor tissue. CD68+ monocytes/macrophages and FoxP3+ regulatory $\mathrm{T}$ cells were scarcely observed, only in the tumors of the PBS control group. Microarray analysis of rUCMSC identified that monocyte chemotactic protein (MCP)-1 is involved in rUCMSCinduced lymphocyte infiltration in the tumor tissues.

Discussion-These results suggest that naïve rUCMSC attenuated mammary tumor growth at least in part by enhancing host anti-tumor immune responses. Thus, naïve UCMSC can be used as powerful therapeutic cells for breast cancer treatment, and MCP-1 may be a key molecule to enhance the effect of UCMSC at the tumor site. 
We have isolated a cell line from the Wharton's jelly of rat umbilical cord, termed rat umbilical cord matrix stem cells (rUCMSC) (1). Characteristics of this rUCMSC are similar to those isolated from human umbilical cord matrix and bone marrow $(2,3)$. Both human and rUCMSC exhibit tumor tropism (4-6). Human UCMSC have proven to be useful as gene delivery vehicles for targeted gene therapy $(4,6)$. We previously discovered that intratumoral (i.t.) or intravenous (i.v.) injection of naïve rUCMSC is capable of causing complete regression of rat mammary tumors in immunocompetent rats (1). Recent studies also indicated that naïve human UCMSC alone possess a strong tumoricidal activity against human breast carcinoma cells in immunodeficient mice $(5,7)$. Both human and rUCMSC have been shown to induce apoptosis and cell cycle arrest in co-cultured tumor cells by UCMSC-produced tumor cell growth regulators $(1,5,7)$. Genome-wide microarray analysis revealed that these growth regulators are potentially associated with multiple tumor suppressor gene products (8) and cell motilityassociated genes (7). These results suggest that naïve UCMSC can be used for cytotherapy for mammary tumors. However, the mechanism by which UCMSC control tumor growth in immunocompetent animals is yet to be completely understood.

The tumor microenvironment, composed of various cell types such as endothelial cells, tumor-infiltrating leukocytes (TILs), fibroblasts and extracellular matrix (ECM), is important in regulating tumor growth (9-13). Fleming et al. (13) have shown that breast ECM has an ability to increase the aggressiveness and tumorigenicity of breast cancer cells. However, a suppressive role for stromal cells in the tumor microenvironment is also reported (14-17). Among components in the tumor microenvironment, TILs play an important role in tumor attenuation $(18,19)$. Particularly, two subsets of lymphocytes, CD8+ T cells and natural killer (NK) cells, are 
suggested to be involved in tumor attenuation by directly damaging tumor cells $(19,20)$. This is supported by the reports that TILs can provide a survival advantage to various cancer patients (20-24). In contrast, regulatory $\mathrm{T}$ (Treg) cells, a subpopulation of $\mathrm{T}$ cells that have the ability to suppress both CD4+ and CD8+ T cell functions (25) indirectly support tumor growth by suppressing tumor-specific CD8+ T cells (26).

Although both naïve human and rUCMSC exhibited strong tumoricidal activity against breast carcinoma cells, a bolus treatment with rUCMSC caused complete tumor regression in immunocompetent rats (1), whereas human UCMSC did not show such powerful tumor growth attenuation effects in an animal study in immunodeficient mice; human UCMSC treatment reduced tumor burden by $50 \%$ (5). These studies prompted us to speculate an involvement of tumor immune response in rUCMSC-dependent strong tumoricidal activity, since immunocompetent animals were used only for the rUCMSC study. In support of this speculation, lymphocyte infiltration in tumor tissues has been shown to be well-correlated with good clinical outcomes in human patients receiving tumor antigen-activated CD8 $+\mathrm{T}$ cell infusions for metastatic melanoma (27).

In the present study, we attempted to clarify the mechanisms by which naïve rUCMSC attenuate/regress tumor growth in vivo and in vitro. Here we report for the first time that treatment with rUCMSC significantly increases lymphocyte infiltration in the tumor tissues, and that TILs, primarily CD8+ T cells and NK cells, induce tumor cell death, thereby regressing tumors. The primary mechanism by which rUCMSC caused significant lymphocyte infiltration in the tumor tissues appears to be due to the increased monocyte chemotactic protein (MCP)-1 secretion from rUCMSC that had homed tumor tissues. 


\section{Materials and Methods}

\section{Cell culture}

rUCMSC were harvested from E19 pregnant rats. The method to isolate and culture rUCMSC was previously described (1). rUCMSC were maintained in low-serum (defined) medium, containing the following mixture per $100 \mathrm{~mL}$ : $57 \mathrm{~mL}$ low-glucose DMEM (Invitrogen, Carlsbad, CA), 37 mL MCBD 201 (Sigma-Aldrich, St. Louis, MO), 2 mL fetal bovine serum (FBS; Atlanta Biologicals, Inc., Lawrenceville, GA), $1 \mathrm{~mL}$ of 100x insulin-transferrin-selenium-X (Invitrogen); $1 \mathrm{~mL}$ of $0.15 \mathrm{~g} / \mathrm{mL}$ AlbuMax I (Invitrogen), $1 \mathrm{~mL}$ of 100 x Pen/Strep (Invitrogen), $10 \mathrm{nmol} / \mathrm{L}$ dexamethasone (Sigma), $100 \mu \mathrm{mol} / \mathrm{L}$ ascorbic acid 2-phosphate (Sigma), $10 \mathrm{ng} / \mathrm{mL}$ epidermal growth factor (R\&D Systems, Minneapolis, MN), and $10 \mathrm{ng} / \mathrm{mL}$ platelet derived growth factor-BB (R\&D Systems). Cells were maintained at $37^{\circ} \mathrm{C}$ in a humidified atmosphere containing 5\% $\mathrm{CO}_{2}$. The rat mammary adenocarcinoma cell line, Mat B III (American Type Culture Collection, Manassas, VA), was maintained in McCoy’s 5A modified medium (Invitrogen) supplemented with 10\% FBS, 100 units $/ \mathrm{mL}$ penicillin, and $100 \mu \mathrm{g} / \mathrm{mL}$ streptomycin (Invitrogen). Cells were cultured at $37^{\circ} \mathrm{C}$ in a humidified atmosphere containing $5 \% \mathrm{CO}_{2}$.

\section{In vivo studies}

All experiments were carried out using protocols approved by the Institutional Animal Care and Use Committee of Kansas State University (\#2681). Female 4 to 5 month-old F344 rats were obtained from Charles River Laboratories (Wilmington, MA). All rats were housed in a clean facility and held for 1 week to acclimatize. On day $0,1 \times 10^{5}$ Mat B III cells suspended in 100 $\mu \mathrm{L}$ of PBS were implanted orthotopically into the abdominal mammary fat pad under isoflurane 
anesthesia. All rats were randomized into two treatment groups: 1) i.t. PBS injection ( $\mathrm{n}=5) ; 2)$ i.t. rUCMSC $\left(1 \times 10^{6}\right)$ transplant $(\mathrm{n}=9)$. On day 4 post-tumor cell transplantation, rUCMSC (1 $\mathrm{x} 10^{6}$ ) suspended in $100 \mu \mathrm{L}$ of PBS were injected i.t. Tumor bearing rats given i.t. $100 \mu \mathrm{L}$ of PBS on day 4 served as control rats. Tumors became palpable starting from day 7; tumors were measured every 3 to 4 days with calipers under isoflurane anesthesia. A portion of rUCMSC treated rats (5 rats) and PBS treated rats were sacrificed 14 days after tumor cell inoculation. Tumor tissues were removed, fixed in $10 \%$ buffered neutral formalin, and used for histological and immunohistochemical analysis. Fresh-frozen tissue was also prepared using O.C.T. Compound (Sakura Finetek USA, Torrance, CA). The remaining 4 rUCMSC-treated rats were used for the monitoring of the tumor size until the tumor size was insignificant.

\section{Microarray analysis}

To examine chemoattractant gene expression, a microarray study was conducted comparing naïve rUCMSC and rUCMSC co-cultured with Mat B III cells. rUCMSC and Mat B III cells were co-cultured using $10 \mathrm{~cm}$ Transwell culture dishes in which the insert membrane pore size is $0.4 \mu \mathrm{m}$ (Corning, Lowell, MA). rUCMSC $\left(1 \times 10^{5}\right)$ were seeded in the bottom culture plate and Mat B III cells $\left(1.5 \times 10^{6}\right)$ were added to the insert on the following day. After 48 hours coculture, total RNAs were isolated using TRIzol Reagent (Invitrogen) following the manufacturer's instructions. Quality and quantity of total RNA samples were assessed using an Agilent 2100 Bioanalyzer (Agilent Technologies, Palo Alto, CA). The microarray analysis, including quality control, was conducted by the Gene Expression and Genomics Unit at the National Institute on Aging (NIA) using RatRef-12 BeadChip (Illumina, San Diego, CA). For each sample, biotinylated cRNAs were prepared using an Illumina total prep RNA amplification 
kit (Applied Biosystems, Foster City, CA, USA). Overall microarray analysis was carried out according to the method described previously (28). Statistical analysis was processed using a Z score developed at NIA (29). Z scores were calculated by subtracting the average gene intensity from the raw intensity data for each gene and dividing that result by the S.D. of all the measured intensities. Gene expression differences between any two experiments were calculated by taking the difference between the observed gene $\mathrm{Z}$ scores. Genes relevant to cell migration, such as chemokines and cytokines, which showed 1.5 times higher expression than in the control group, were selected for the final gene list.

\section{Cell migration assay}

Migration of peripheral blood mononuclear cells (PBMC) was carried out using Transwell cell culture plates with $5 \mu \mathrm{m}$ pores (Corning) according to the previously described method (30). The bottom membrane of the Transwell inserts was coated with Matrigel $(200 \mu \mathrm{g} / \mathrm{ml})$ before use. In the bottom of the Transwell culture plate, $3 \times 10^{4} \mathrm{rUCMSC}$ or Mat B III cells were seeded and cultured for 24 hrs. PBMC $\left(0.5 \times 10^{5}\right)$ isolated from the peripheral blood obtained from an adult female F344 rat were suspended in $100 \mu 1$ of defined medium and added to the Transwell insert. Antibodies against MCP-1(Santa Cruz Biotechnology, Santa Cruz, CA) or control IgG were added in the lower chamber at the concentration of 2 or $4 \mu \mathrm{g} / \mathrm{well} 3 \mathrm{hrs}$ prior to the addition of PBMC. Cells were incubated at $37^{\circ} \mathrm{C}$ for $3 \mathrm{hrs}$. At the end of the incubation, the membrane of the Transwell insert was fixed in methanol, stained with Giemsa solution for $15 \mathrm{~min}$, and dried overnight. The membrane was removed from the insert and embedded in Permount (Fisher Scientific, Pittsburgh, PA). The number of the PBMCs stained with Giemsa solution on the underside of the membrane (i.e., is the side facing the rUCMSC or Mat B III cells in the bottom 
chamber) was counted under the microscope.

\section{The comparison of the expression of MCP-1 in rUCMSC and Mat B III by Western blot} analysis

Western blot analysis was conducted to examine chemoattractant protein expression. rUCMSC and Mat B III cells were co-cultured using 6-well Transwell culture plates in which the insert membrane pore size is $0.4 \mu \mathrm{m}$ (Corning). $\mathrm{rUCMSC}\left(5 \times 10^{3}\right)$ were seeded in the bottom culture plate and Mat B III cells $\left(7.5 \times 10^{4}\right)$ were added to the insert on the following day. After 72 hours co-culture, total cellular protein of rUCMSC or Mat B III was prepared using RIPA buffer (Sigma). Protein samples were separated by $12 \%$ SDS-PAGE, electroblotted onto nitrocellulose membrane (GE Healthcare, Uppsala, Sweden) and blocked with 4\% nonfat dry milk in $0.1 \%$ Tween 20 in phosphate buffered saline (PBST) for $1 \mathrm{hr}$ at room temperature. The membranes were washed and incubated with antibody against MCP-1 (1:200, Santa Cruz Biotechnology) with 4\% nonfat dry milk in PBST for $1 \mathrm{hr}$ at room temperature, followed by incubation with a horseradish peroxidase-conjugated anti-rabbit IgG secondary antibody (1: 2000, GE Healthcare) for $1 \mathrm{hr}$ at room temperature. The protein expression signal was detected with SuperSignal West Femto Maximum Sensitivity Substrate (Pierce, Rockford, IL). GAPDH was used as the loading control of sample by reprobing with an anti-GAPDH antibody (1:2000, Santa Cruz Biotechnology).

\section{Histological analysis}

Paraffin-embedded mammary tumor tissues were sectioned at $4 \mu \mathrm{m}$ and stained with hematoxylin and eosin for histological examination. Some unstained sections were selected for 
immunohistochemistry to determine the $\mathrm{CD}$ antigens on the lymphocytes in the tumor tissues. Slides were deparaffinized and rehydrated before staining. The heat-induced antigen unmasking was performed in citrate buffer for 5 minutes using an autoclave. Sections were then incubated with $3 \%$ hydrogen peroxide in methanol for 3 minutes to block endogenous peroxide activity. For CD4 and Foxp3 immunostaining, fresh-frozen tissue sections $(4 \mu \mathrm{m})$ fixed by acetone were prepared and incubated in 4\% non-fat skim milk in PBS at room temperature for 20 minutes. The dilution of antibodies for CD3 (Dako North America, Carpinteria, CA), CD8 (BD Biosciences, Rockville, MD), CD20 (Santa Cruz Biotechnology) and CD68 (Santa Cruz Biotechnology) was 1:50. The dilution of antibodies for CD4 (AbD Serotec, Raleigh, NC), Foxp3 (Aviva System Biology, San Diego, CA) and NK cell marker (Santa Cruz Biotechnology) was 1:100. Sections were incubated with the primary antibodies for 60 minutes at room temperature. For immunostaining for CD4 and CD20, sections were incubated with biotin-conjugated antibody against mouse IgG and goat IgG (Vector Laboratories, Burlingame, CA), respectively, followed by reaction with the avidin-biotin peroxidase complex $(\mathrm{ABC})$ reagent (Vector Laboratories) for 40 minutes at room temperature. For immunostaining for CD3, CD8 and CD68, Foxp3 and NK cell marker, sections were incubated with biotin-conjugated antibody against rabbit IgG (Vector Laboratories) before reaction with the $\mathrm{ABC}$ reagent (Vector Laboratories). Peroxidase activity was visualized with 3, 3'-diaminobenzodine tetrahydrochloride (Sigma). Sections were lightly counterstained with Mayer's hematoxylin. The average number of each cell types (bar graphs) was determined by analyzing ten tumor areas in each treatment group and was expressed as cell number per high power (400x) field.

\section{Statistical analysis}


All data are reported as mean \pm SE. Data were analyzed by Student $t$ test. Group comparisons were deemed significant for 2-tailed P values below 0.05 .

\section{Results}

\section{rUCMSC treatment significantly attenuates the growth of mammary tumor grafts in vivo}

I.t. injection of rUCMSC significantly attenuated the growth of orthotopic grafts of rat mammary tumors as compared with those of PBS-injected control rats (sham, Fig. 1A). The tumor growth reached a peak at the 16-19 days after tumor inoculation and regressed thereafter. Tumor size was insignificant 40 days after tumor cell inoculation. In PBS-injected control rats, the tumors grew continuously; rats were sacrificed 14 days after tumor inoculation. To evaluate leukocyte infiltration in the tumor tissues, a portion of rats were sacrificed at 14 days after tumor cell inoculation and tumor tissues were removed for immunohistochemical analysis. The tumor weights measured on day 14 showed a significant difference between rUCMSC- and PBSinjected groups (Fig. 1B).

\section{rUCMSC treatment stimulated infiltration of numerous lymphocytes into the tumor}

As shown in Fig. 2A, PBS-treated orthotopic tumors in the mammary fat pad were composed of solidly packed round to oval cells. All tumor cells had irregularly-shaped and-sized nuclei with prominent nucleoli. A large number of mitotic figures were also commonly observed. rUCMSC treatment significantly increased the amount of ECM deposition and decreased the number of tumor cells within the tumor tissues (Fig. 2B). In addition, rUCMSC treatment caused infiltration of numerous lymphocytes, but only a small number of neutrophils, in the peritumoral area of the tumor mass. This leukocyte infiltration was not observed in the PBS control group (Fig. 2A). 
rUCMSC treatment increased the infiltration of CD4+ and CD8+ T cells and NK cells and decreased macrophages and Treg cells in the tumor

To identify TILs populations, immunohistochemical analysis was performed using antibodies against CD3 (T cells), CD20 (B cells), CD4 (helper T cells), CD8 (cytotoxic T cells), Foxp3 (Treg) and CD68 (monocytes/macrophages). As shown in Fig. 3, rUCMSC treatment caused a 3 fold increase of CD3+ cells (average 150 cells/field) over the PBS-treated control (average 50 cells/field). Treatment with rUCMSC also caused a 3 fold increase of CD20+ cells (average 30 cells/field) over the control tumors. However, CD20+ cells constituted only $20 \%$ of total lymphocytes infiltrating the tumor tissue after the treatment, indicating that the main component of lymphocytes in the tumor tissue was T cells rather than B cells (Fig. 3). In the subpopulation of T cells, treatment with rUCMSC significantly increased the number of both CD4+ (3 fold) and CD8+ cells (2 fold, Fig. 3). The average number of CD8+ cells was higher than that of CD4+ cells. On the contrary, a small number of Foxp3+ cells were observed only in the PBS group, but not in the rUCMSC treated group (Fig. 3). Although NK cells were not a major component of theTILs, NK cells were also significantly higher (1.8 fold higher) in the rUCMSC treated group than in the control group. Interestingly, CD68+ cells were infrequently observed only in the PBS group, but not in the tumors treated with rUCMSC (Fig. 3). These results indicate that rUCMSC treatment caused the tumor tissue infiltration of helper and cytotoxic T cells and NK cells, but decreased Treg cells and monocytes/macrophages.

\section{Microarray analysis of cytokines related for cell migration}

Gene expression of rUCMSC co-cultured with Mat B III was compared with naïve rUCMSC. A 
partial list of the highly up- or down-regulated chemokines, cytokines and immune cell migration-related genes which showed more than 1.5 times difference in Z-ratio is presented in Table 2. Notably, expression of MCP-1 was significantly increased in rUCMSC (13.88 fold) when they were co-cultured with Mat B III cells. Since MCP-1 is a one of the chemoattractants associated with lymphocyte infiltration, MCP-1 is a potential candidate for stimulating lymphocyte migration into the rUCMSC-treated tumors.

\section{MCP-1 expression is increased in rUCMSC when they were co-cultured with Mat B III cells}

To confirm the above mRNA expression results from microarray analysis in protein levels, the expression of MCP-1 was semi-quantified by Western blot analysis. As shown in Fig. 4, the basal expression of MCP-1 in rUCMSC was significantly larger than that in Mat B III. Furthermore, the expression of the MCP-1 in rUCMSC was significantly increased when they were cocultured with Mat B III cells. These results suggest that MCP-1 expression is sensitively upregulated in response to the presence of Mat B III mammary carcinoma cells.

\section{rUCMSC stimulated PBMC migration by MCP-1 secretion}

To examine whether PBMC migrate toward rUCMSC and whether this migration is controlled by MCP-1, PBMC migration was examined by the Boyden chamber assay using Transwell culture system in the presence or absence of MCP-1 neutralizing antibody. As shown in Fig. 5, rUCMSC increased approximately two -fold the infiltration of PBMC into the Matrigel coated membrane of the Transwell insert. The number of migrating PBMC was significantly decreased by adding $4 \mu \mathrm{g} / \mathrm{well}$ of neutralizing antibody against MCP-1, indicating that the secretion of 
MCP-1 from rUCMSC is an important factor for migration of PBMC, which are mainly lymphocytes. In the control study, the treatment of Mat B III with neutralizing antibody against MCP-1 did not show much effect on the PBMC infiltration to the bottom of the Transwell membrane. This result suggests that PBMC migration toward rUCMSC is specific. In the control experiment with anti-GFP antibody instead of MCP-1 antibody, the number of the membraneinfiltrating PBMC was slightly higher than that without any antibody, but no statistically significant difference was observed. These results suggest that the effect of the MCP-1 antibody is specific and PBMC specifically migrate toward rUCMSC by sensing the MCP-1gradient.

\section{Discussion}

It has been shown that stem cells are useful therapeutic tools for various diseases including cancers. UCMSC are unique $\operatorname{MSC}(2,3)$ and are considered to be safe therapeutic cells, since systemic or subcutaneous administration of UCMSC does not result in adverse effects or tumor formation in the recipients $(4,31)$. In preclinical animal studies involving the use of naïve UCMSC for cancer treatment, rUCMSC have demonstrated a remarkable ability to cause complete regression of Mat B III rat mammary tumors in vivo (1). In addition, unengineered human UCMSC have been shown to attenuate human breast cancer xenografts in a SCID mouse model (5). However, the mechanism by which naïve UCMSC attenuate tumor growth has yet to be fully understood. Although previous studies have demonstrated that diffusible factors produced by naïve UCMSC play important roles in attenuation of the growth of tumor cells in vitro, the nature of factors and the mechanisms of in vivo tumor growth attenuation are yet to be clarified. Therefore, the present study was focused on clarifying the 
mechanism by which rUCMSC attenuate the growth of mammary tumors both in vitro and in an in vivo rat model. The present study shows that rUCMSC enhance host anti-tumor immune responses by recruiting primarily $\mathrm{CD} 4+$ and $\mathrm{CD} 8+\mathrm{T}$ cells and NK cells to tumor tissues, thus significantly attenuating mammary tumor growth. The results further suggest that a potential mechanism by which naïve rUCMSC increase lymphocyte infiltration into the tumor tissues is due to the increase of MCP-1 secretion.

The present study showed that treatment with naive rUCMSC significantly attenuated the growth of orthotopic rat mammary tumors (Fig. 1). This is consistent with the previous reports (1), in which a single treatment with rUCMSC completely abolished rat mammary tumors. Immunohistochemical analysis of tumor tissues treated with rUCMSC revealed a significant increase of lymphocyte infiltration in the tumor tissues (Fig. 2). Detailed analysis further revealed that the major component of infiltrating lymphocytes in rUCMSC-treated tumor tissues is CD8+ cells (approximately $52 \%$ of total lymphocytes are CD8+ T cells and $37.5 \%$ cells correspond to CD4+ T cells, Fig. 3). Although CD20+ cells were also increased in the rUCMSC treated tumors, CD20+ cells comprised less than $12 \%$ of total lymphocytes (Fig. 3). In addition, a unique discovery in the present study was that no CD68+ or Foxp3+ cells were detected in tumor tissues in rUCMSC-treated mice (Fig. 3E), although a small number of CD68+ and Foxp3+ cells were consistently detected in untreated tumors. These results strongly suggest that treatment with naïve rUCMSC significantly enhanced host tumor immune responses by increasing CD8+ cytotoxic T cells and decreasing CD68+ macrophages and Foxp3+ Treg cells. In a variety of human cancers, it has been shown that TILs, such as cytotoxic T cells (CD8+), helper T cells (CD4+), and Treg cells (Foxp3+) play important roles in controlling tumor growth (26). Among those TILs CD8+ T cells are the major subset of T cells, and they can become 
tumor-specific cytotoxic cells by direct recognition of tumor antigens presented on the tumor cells (24). Infiltration of CD8+ T cells in the tumor site is shown to be associated with a good prognosis in malignant tumors such as colorectal, ovarian and esophageal carcinoma (20-23). These reports support the idea that rUCMSC-dependent tumor growth attenuation is associated with stimulation of host anti-tumor immune responses via $\mathrm{CD} 8+\mathrm{T}$ cell infiltration in the tumor tissues. Furthermore, an increase of CD4+ helper T cells in rUCMSC-treated tumors (Fig. 3) further supports the idea of stimulation of host anti-tumor immune responses, since helper $\mathrm{T}$ cells assist in activation and proliferation of cytotoxic T cells (32). Decrease of Treg cells also supports an increase of cytotoxic $\mathrm{T}$ cells, since they directly attenuate $\mathrm{T}$ cell proliferation (33). Indeed, therapies to decrease the Treg cell population or to blunt the function of Treg cells are effective in attenuating various tumors (34). In contrast, numerous reports indicate an increase of Treg cells in tumor tissues correlates with tumor progression and poor prognosis of multiple organ type cancers including breast cancer (35).

In the present study, rUCMSC treatment also increased NK cells in tumor tissues (Fig. 3). Since NK cells are an important component of anti-tumor immune reactions in addition to cytotoxic T cells, an increase of NK cells enhances anti-tumor immune reactions. Although NK cells are innate immune cells and do not have the specificity to particular tumors, they quickly induce apoptosis in tumor cells thereby attenuating tumor burden (36). Taken together, these results clearly indicate that treatment with rUCMSC increases the infiltration of the CD8+ T cells and NK cells into tumor tissues and decreases CD68+ macrophages and Foxp3+ Treg cells, thereby reducing the tumor volume. The granzyme-positive population in the CD8+ T cells in the tumor tissues should be clarified in future studies. 
Our results from the present study indicate that rat PBMC migration is directed toward rUCMSC rather than Mat B III mammary carcinoma cells (Fig. 5). This migration is associated with MCP-1, as MCP-1 neutralizing antibody attenuates PBMC migration to the rUCMSC (Fig. 5). This is directly relevant to the histological findings showing that i.t. injection of rUCMSC induced infiltration of numerous lymphocytes into the tumor tissues (Figs. 2 and 3). MCP-1 is shown to play an important role in migration of lymphocytes and NK cells to tumor tissues. MCP-1 is a member of the chemokine-2 subfamily that attracts and activates monocytes or lymphocytes at subnanomolar concentrations (37-39). Expression of MCP-1 in tumor tissues is shown to be sufficient for induction of cytotoxic $\mathrm{T}$ cell migration to tumor tissues in mouse xenograft studies (40). Tsuchiyama et al. (41) have reported that a therapy with recombinant adenovirus vector (rAd) expressing suicide gene, HSV thymidine kinase, and MCP-1 completely eradicated hepatocellular carcinoma by the recruitment of NK cells. The present study suggests that rUCMSC traffic to tumor tissues and recruit $\mathrm{T}$ cells to the tumor site through increased MCP-1 secretion. To the best of our knowledge, this study is the first report showing that rUCMSC secrete a chemoattractant such as MCP-1 and induce lymphocyte infiltration. Hence, this report suggests that MCP-1 could be a potential therapeutic target to enhance local immune responses to attenuate mammary carcinoma.

Interestingly, CD68+ cells were not observed in the rUCMSC-treated tumors despite the fact that CD68+ macrophages are known to be attracted by MCP-1 (42). The likely explanation is that a factor inhibitory to macrophages might be secreted from rUCMSC or Mat B III cells. Indeed, the microarray analysis indicated that the expression of macrophage inhibitory factor (MIF) was significantly increased (3.4 fold) in rUCMSC co-cultured with Mat B III cells (Table 1). Therefore, it is likely that increased MIF expression may have stimulated an exodus of 
macrophages from the rUCMSC-treated tumors, thus assisting rUCMSC-dependent attenuation of tumor growth. Clarification of the role of MIF in rUCMSC-treated tumors requires further studies.

Regarding macrophage infiltration in the tumor tissues, clinical studies have sought to identify correlations between macrophage density and prognosis. A summary in a review by Bingle et al. (43) using a meta-analysis showed that in $>80 \%$ of the cases, an increased macrophage density in tumor tissues was associated with poor prognosis, and the remaining $20 \%$ of cases were split between having no effect and those with good prognosis. This was confirmed with simple histological evaluation of macrophage density, which showed that macrophage density in itself was an independent predictor of poor outcome $(44,45)$. In consideration of these reports, the microenvironment without macrophages results in a better probability for attenuating tumor growth. However, further experiments are required to clarify the relationship between the decrease of macrophage density and the effect of rUCMSC on tumor growth attenuation.

Among many tissue-originated multipotent stem cells, UCMSC are very usable due to unethical abundant resources, the simplicity of their preparation method (46), bankability (47), low immunogenicity $(48,49)$, and tropism to inflammatory tissues such as tumor tissues $(1,4,6)$ etc. In particular, human UCMSC apparently escape the host immune responses by multiple mechanisms (8); they express MHC class I but not class II antigens; they are negative for other co-stimulatory antigens such as CD80 and CD86 $(50,51)$; they produce large amounts of tolerogenic interleukin-10 and transforming growth factor- $\beta$ and express human leukocyte antigen G (HLA-G) at higher levels than bone marrow derived mesenchymal stem cells (50-52). This poor immunogenicity allows human UCMSC to be usable for allogeneic transplantation in potential human applications. In relation to this poor immunogenicity, it is noteworthy to cite 
that Chao et al. have reported a successful transplantation of human UCMSC-derived islet-like cells in diabetic rats without using immunosuppressants (53). The tropism of UCMSC to the inflammatory tissues, such as tumor tissues, make this cell also usable as targeted drug or gene delivery vehicles. Recent reports have shown that human UCMSC engineered to express interferon (IFN)- $\beta$, which is a strong apoptosis inducer, shown to be migrated to metastatic human breast tumor nodules in the lung (4) or to the bronchioloalveolar carcinoma nodules (6), produced sufficient amounts of the IFN- $\beta$ in the tumor tissues and significantly attenuated tumor growth in mice. Therefore, human UCMSC engineered to express MCP-1 are expected to migrate to the tumor tissues, recruit a large numbers of CD8+, CD4+ T cells and NK cells to the tumor sites, and control tumor growth. To ensure these potential applications, further studies are needed to confirm above mentioned possibilities. Although a live cell therapy with multipotent stem cells holds a significantly possibilities, a potential limitation of this cell therapy could be a migration to unexpected tissues and differentiations. Therefore, safety of live cell therapy and fate of multipotent stem cells in human body should be carefully clarified in the future.

In conclusion, bolus treatment with naive rUCMSC significantly attenuated the growth of orthotopic rat mammary carcinoma grafts. This tumor growth attenuation is associated with a marked increase of CD8+ and CD4+ T cell and NK cell infiltration and escape of CD68+ macrophages and Foxp3+ Treg cells from tumor tissues. Recruitment of T cells and NK cells and an escape of CD68+ cells appear to be mediated by MCP-1 and MIF, respectively, and they were secreted mainly from the rUCMSC. Thus, the rUCMSC traffic to tumor tissues, recruit cytotoxic lymphocytes by the secretion of lymphocyte migration cytokines such as MCP-1 and MIF, thereby controlling tumor growth. UCMSC-based cytotherapy may be a new therapeutic modality for the treatment of mammary carcinoma. 


\section{Acknowledgements}

We are grateful to Mr. William H. Wood III (National Institute on Aging, NIH) for his excellent technical assistance in microarray analysis. We are thankful to Mr. Sivasai Balivada (Department of Anatomy \& Physiology, Kansas State University) for his technical assistance in animal studies. This work was supported by Kansas State University (KSU) Terry C. Johnson Center for Basic Cancer Research, Kansas Bioscience Authority Collaborative Cancer Research Initiative grant, Kansas State Legislative Appropriation, KSU College of Veterinary Medicine Dean's fund, NIH grants P20 RR017686, P20 RR016475, P20 RR01556, R21 CA135599 and the Intramural

Research Program of the NIH, National Institute on Aging.

\section{References}

1. Ganta C, Chiyo D, Ayuzawa R, Rachakatla R, Pyle M, Andrews G, et al. Rat umbilical cord stem cells completely abolish rat mammary carcinomas with no evidence of metastasis or recurrence 100 days post-tumor cell inoculation. Cancer Res. 2009;69:1815-20.

2. Weiss ML, Medicetty S, Bledsoe AR, Rachakatla RS, Choi M, Merchav S, et al. Human umbilical cord matrix stem cells: preliminary characterization and effect of transplantation in a rodent model of Parkinson's disease. Stem Cells. 2006;24:781-92.

3. Troyer DL, Weiss ML. Wharton's jelly-derived cells are a primitive stromal cell population. Stem Cells. 2008;26:591-9.

4. Rachakatla RS, Marini F, Weiss ML, Tamura M, Troyer D. Development of human umbilical cord matrix stem cell-based gene therapy for experimental lung tumors. Cancer Gene Ther. 2007;14:828-35.

5. Ayuzawa A, Doi C, Rachakatla RS, Pyle MM, Maurya DK, Troyer D, et al. Naïve human umbilical cord matrix derived stem cells significantly attenuate growth of human breast cancer cells in vitro and in vivo. Cancer letters. 2009;280:31-7.

6. Matsuzuka T, Rachakatla RS, Doi C, Maurya DK, Ohta N, Kawabata A, et al. Human umbilical cord matrix-derived stem cells expressing interferon-beta gene significantly attenuate bronchioloalveolar carcinoma xenografts in SCID mice. Lung Cancer. 2010;70:28-36.

7. Chao KC, Yang HT, Chen MW. Human umbilical cord mesenchymal stem cells suppress breast cancer tumorigenesis through direct cell-cell contact and internalization. J Cell Mol Med. 2012;16:1803-15.

8. Tamura M, Kawabata A, Ohta N, Uppalapati L, Becker K, Troyer D. Wharton's Jelly Stem Cells as Agents for Cancer Therapy. Open Tissue Eng Regen Med J. 2011;4:39-47. 
9. van Roozendaal KE, Klijn JG, van Ooijen B, Claassen C, Eggermont AM, Henzen-Logmans SC, et al. Differential regulation of breast tumor cell proliferation by stromal fibroblasts of various breast tissue sources. Int J Cancer. 1996;65:120-5.

10. Barcellos-Hoff MH, Ravani SA. Irradiated mammary gland stroma promotes the expression of tumorigenic potential by unirradiated epithelial cells. Cancer Res. 2000;60:1254-60.

11. Shekhar MP, Werdell J, Santner SJ, Pauley RJ, Tait L. Breast stroma plays a dominant regulatory role in breast epithelial growth and differentiation: implications for tumor development and progression. Cancer Res. 2001;61:1320-6.

12. Sadlonova A, Novak Z, Johnson MR, Bowe DB, Gault SR, Page GP, et al. Breast fibroblasts modulate epithelial cell proliferation in three-dimensional in vitro co-culture. Breast Cancer Res. 2005;7:R46-59.

13. Fleming JM, Miller TC, Quinones M, Xiao Z, Xu X, Meyer MJ, et al. The normal breast microenvironment of premenopausal women differentially influences the behavior of breast cancer cells in vitro and in vivo. BMC Med. 2010;8:27.

14. Karlan BY, Baldwin RL, Cirisano FD, Mamula PW, Jones J, Lagasse LD. Secreted ovarian stromal substance inhibits ovarian epithelial cell proliferation. Gynecol Oncol. 1995;59:67-74.

15. Dong-Le Bourhis X, Berthois Y, Millot G, Degeorges A, Sylvi M, Martin PM, et al. Effect of stromal and epithelial cells derived from normal and tumorous breast tissue on the proliferation of human breast cancer cell lines in co-culture. Int J Cancer. 1997;71:2-8.

16. Arnold JT, Lessey BA, Seppälä M, Kaufman DG. Effect of normal endometrial stroma on growth and differentiation in Ishikawa endometrial adenocarcinoma cells. Cancer Res. 2002;62:79-88.

17. Proia DA, Kuperwasser C. Stroma: tumor agonist or antagonist. Cell Cycle. 2005;8:1022-5.

18. Disis ML, Bernhard H, Jaffee EM. Use of tumour-responsive T cells as cancer treatment. Lancet. 2009;373:673-83.

19. Kasper HU, Drebber U, Stippel DL, Dienes HP, Gillessen A. Liver tumor infiltrating lymphocytes: comparison of hepatocellular and cholangiolar carcinoma. World J Gastroenterol. 2009;15:5053-7.

20. Schumacher K, Haensch W, Röefzaad C, Schlag PM. Prognostic significance of activated CD8(+) T cell infiltrations within esophageal carcinomas. Cancer Res. 2001;61:3932-6.

21. Zhang L, Conejo-Garcia JR, Katsaros D, Gimotty PA, Massobrio M, Regnani G, et al. Intratumoral T cells, recurrence, and survival in epithelial ovarian cancer. $\mathrm{N}$ Engl J Med. 2003;348:203-13.

22. Menon AG, Jansen-van Rhijin C, Morreau H, Putter H, Tollenaar RA, van de Velde CJ, et al. Immune system and prognosis in colorectal cancer: a detailed immunohistochemical analysis. Lab Invest. 2004;84:493-501.

23. Prall F, Dührkop T, Weirich V, Ostwald C, Lenz P, Nizze H, et al. Prognostic role of CD8+ tumor-infiltrating lymphocytes in stage III colorectal cancer with and without microsatellite instability. Hum Pathol. 2004;35:808-16.

24. Ryschich E, Cebotari O, Fabian OV, Autschbach F, Kleeff J, Friess H, et al. Loss of heterozygosity in the HLA class I region in human pancreatic cancer. Tissue Antigens. 2004;64:696-702.

25. Jonuleit H, Schmitt E, Stassen M, Tuettenberg A, Knop J, Enk AH. Identification and functional characterization of human $\mathrm{CD} 4(+) \mathrm{CD} 25(+) \mathrm{T}$ cells with regulatory properties isolated from peripheral blood. J Exp Med. 2001;193:1285-94.

26. Curtin JF, Candolfi M, Fakhouri TM, Liu C, Alden A, Edwards M, et al. Treg depletion inhibits efficacy of cancer immunotherapy: implications for clinical trials. PLoS One. 2008;3:e1983.

27. Pockaj BA, Sherry RM, Wei JP, Yannelli JR, Carter CS, Leitman SF, et al. Localization of 111 indium-labeled tumor infiltrating lymphocytes to tumor in patients receiving adoptive immunotherapy. Augmentation with cyclophosphamide and correlation with response. Cancer. 1994;73:1731-7. 
28. Li J, Chigurupati S, Agarwal R, Mughal MR, Mattson MP, Becker KG, Wood WH 3rd, Zhang Y, Morin PJ. Possible angiogenic roles for claudin-4 in ovarian cancer. Cancer Biol Ther. 2009;8:1806-14.

29. Cheadle C, Vawter MP, Freed WJ, Becker KG. Analysis of microarray data using Z score transformation. J Mol Diagn. 2003;5:73-81.

30. Tsai WH, Shih CH, Lin CC, Ho CK, Hsu FC, Hsu HC. Monocyte chemotactic protein-1 in the migration of differentiated leukaemic cells toward alveolar epithelial cells. Eur Respir J. 2008;31:957-62.

31. Rachakatla RS, Pyle MM, Ayuzawa R, Edwards SM, Marini FC, Weiss ML, et al. Combination treatment of human umbilical cord matrix stem cell-based interferon-beta gene therapy and 5fluorouracil significantly reduces growth of metastatic human breast cancer in SCID mouse lungs. Cancer Invest. 2008;26:662-70.

32. Abbas AK, Murphy KM, Sher A. Functional diversity of helper T lymphocytes. Nature. 1996;383:787-93.

33. Woo EY, Yeh H, Chu CS, Schlienger K, Carroll RG, Riley JL, et al. Cutting edge: Regulatory T cells from lung cancer patients directly inhibit autologous $\mathrm{T}$ cell proliferation. J Immunol. 2002;168:4272-6.

34. Colombo MP, Piconese S. Regulatory-T-cell inhibition versus depletion: the right choice in cancer immunotherapy. Nat Rev Cancer. 2007;7:880-7.

35. Beyer M, Schultze JL. Regulatory T cells in cancer. Blood. 2006;108:804-11.

36. Zamai L, Ponti C, Mirandola P, Gobbi G, Papa S, Galeotti L, et al. NK cells and cancer. J Immunol. 2007;178:4011-6.

37. Leonard EJ, Yoshimura T. Human monocyte chemoattractant protein-1 (MCP-1). Immunol Today. 1990;11:97-101.

38. Oppenheim JJ, Zachariae CO, Mukaida N, Matsushima K. Properties of the novel proinflammatory supergene "intercrine" cytokine family. Annu Rev Immunol. 1991;9:617-48.

39. Carr MW, Roth SJ, Luther E, Rose SS, Springer TA. Monocyte chemoattractant protein 1 acts as a T-lymphocyte chemoattractant. Proc Natl Acad Sci U S A. 1994;91:3652-6.

40. Brown CE, Vishwanath RP, Aguilar B, Starr R, Najbauer J, Aboody KS, et al. Tumor-derived chemokine MCP-1/CCL2 is sufficient for mediating tumor tropism of adoptively transferred $\mathrm{T}$ cells. J Immunol. 2007;179:3332-41.

41. Tsuchiyama T, Nakamoto Y, Sakai Y, Marukawa Y, Kitahara M, Mukaida N, et al. Prolonged, NK cell-mediated antitumor effects of suicide gene therapy combined with monocyte chemoattractant protein-1 against hepatocellular carcinoma. J Immunol. 2007; 178:574-83.

42. Maus UA, Waelsch K, Kuziel WA, Delbeck T, Mack M, Blackwell TS, et al. Monocytes are potent facilitators of alveolar neutrophil emigration during lung inflammation: role of the CCL2CCR2 axis. J Immunol. 2003;170:3273-8.

43. Bingle L, Brown NJ, Lewis CE. The role of tumor-associated macrophages in tumor progression: implications for new anticancer therapies. J Pathol. 2002;196:254-65.

44. Dave SS, Wright G, Tan B, Rosenwald A, Gascoyne RD, Chan WC, et al. Prediction of survival in follicular lymphoma based on molecular features of tumor-infiltrating immune cells. N. Engl. J. Med. 2004;351:2159-69.

45. Farinha P, Masoudi H, Skinnider BF, Shumansky K, Spinelli JJ, Gill K, et al. Analysis of multiple biomarkers shows that lymphoma-associated macrophage (LAM) content is an independent predictor of survival in follicular lymphoma (FL). . Blood. 2005; 106:2169-74.

46. Mitchell KE, Weiss ML, Mitchell BM, Martin P, Davis D, Morales L, et al. Matrix cells from Wharton's jelly form neurons and glia. Stem Cells. 2003;21:50-60.

47. Gong W, Han Z, Zhao H, Wang Y, Wang J, Zhong J, et al. Banking human umbilical cordderived mesenchymal stromal cells for clinical use. Cell Transplant. 2012;21:207-16.

48. Cho PS, Messina DJ, Hirsh EL, Chi N, Goldman SN, Lo DP, et al. Immunogenicity of umbilical cord tissue derived cells. Blood. 2008;111:430-8. 
49. Weiss ML, Anderson C, Medicetty S, Seshareddy KB, Weiss RJ, VanderWerff I, et al. Immune properties of human umbilical cord Wharton's jelly-derived cells. Stem Cells. 2008;26:2865-74.

50. La Rocca G, Anzalone R, Corrao S, Magno F, Loria T, Lo Iacono M, et al. Isolation and characterization of Oct-4+/HLA-G+ mesenchymal stem cells from human umbilical cord matrix: differentiation potential and detection of new markers. Histochem Cell Biol. 2009;131:267-82.

51. Prasanna SJ, Gopalakrishnan D, Shankar SR, Vasandan AB. Pro-inflammatory cytokines, IFNgamma and TNFalpha, influence immune properties of human bone marrow and Wharton jelly mesenchymal stem cells differentially. PLoS One. 2010;5:e9016.

52. Deuse T, Stubbendorff M, Tang-Quan K, Phillips N, Kay MA, Eiermann T, et al. Immunogenicity and immunomodulatory properties of umbilical cord lining mesenchymal stem cells. Cell Transplant. 2011;20:655-667.

53. Chao KC, Chao KF, Fu YS, Liu SH. Islet-like clusters derived from mesenchymal stem cells in Wharton's Jelly of the human umbilical cord for transplantation to control type 1 diabetes. PLoS One. 2008;3:e1451. 
Table 1. Microarray analysis of rUCMSC and those co-cultured with Mat B III mammary tumor cell

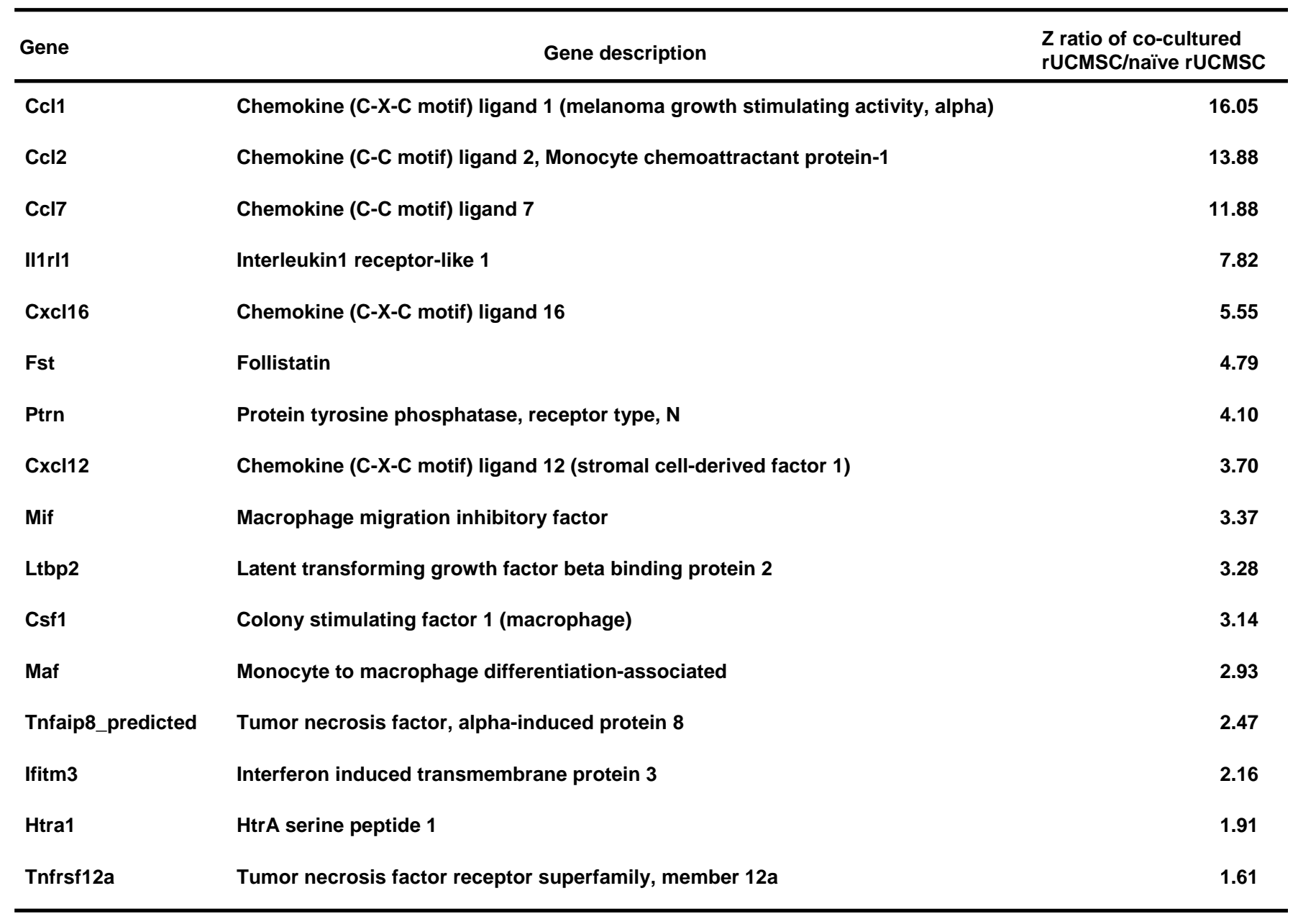




\section{Figures}

Figure 1. Bolus injection of naïve rUCMSC in tumors significantly suppressed growth of orthotopic Mat B III autografts in F344 rats.

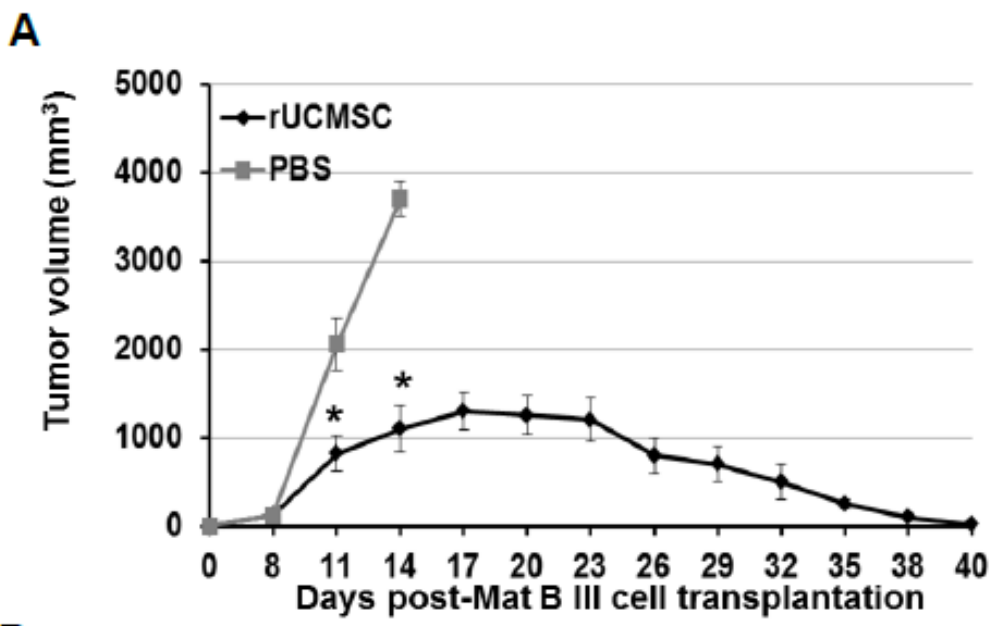

B

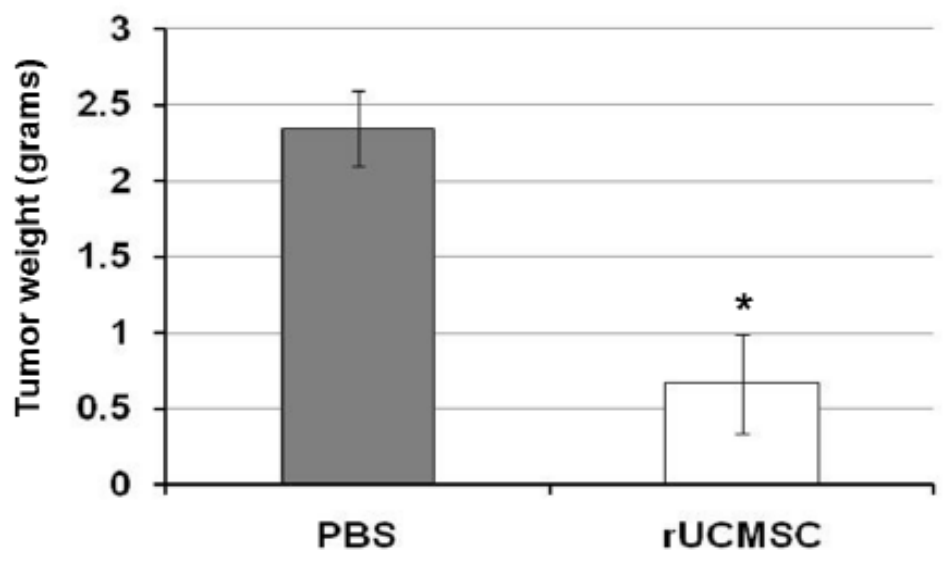

Figure 1. Bolus injection of naïve rUCMSC in tumors completely attenuated the growth of orthotopic Mat B III grafts in a syngeneic F344 rat model. A single i.t. administration of rUCMSC (1 million cells, $n=9$ ) significantly attenuated the Mat B III tumor growth curve (1 million Mat B III cells) and completely regressed the tumors at day 40 (panel A). Five each of rUCMSC- or PBS- treated rats were sacrificed 14 days after tumor cell inoculation. The average tumor weight in rUCMSC-treated rats was significantly smaller than that in PBS-treated rats $\left(n=5\right.$, panel B). ${ }^{*}, P<0.05$ compared with PBS-treated control. 
Figure 2. Mononuclear leukocyte infiltration was significantly increased in rUCMSC treated orthotopic Mat B III grafts.

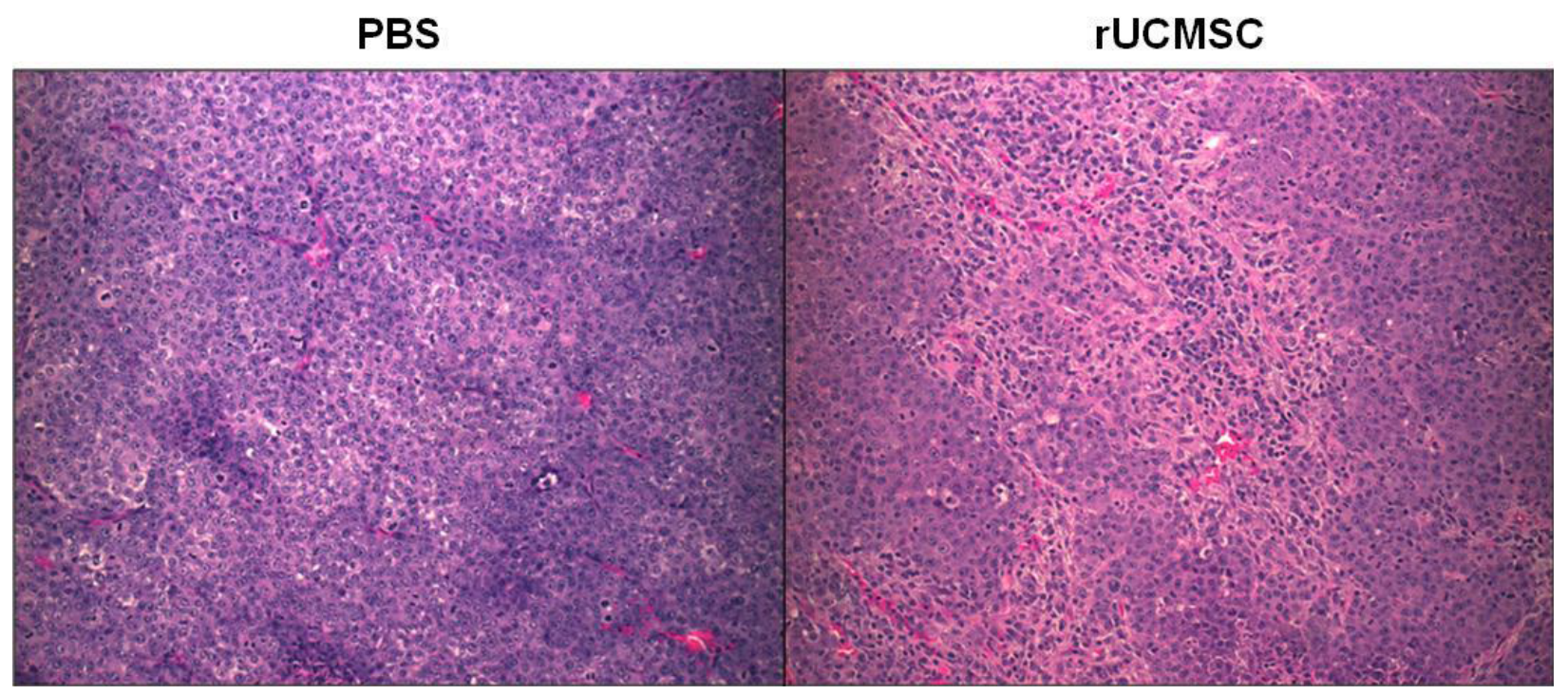

Figure 2. Mononuclear leukocyte infiltration was significantly increased in rUCMSC-treated orthotopic Mat B III grafts. Samples were prepared as described in Materials and Methods and subjected to histological analysis after H\&E staining. The pictures represent typical PBS- and rUCMSC-treated tumors. As shown in panel A, a majority of cells in the PBS-treated tumors are tumor cells (inset), whereas panel $B$ shows that a large portion in the tumor was composed of connective tissues (areas with pinkish color) and a large number of mononuclear cells (inset). Scale bars $=200 \mu \mathrm{m}$. 


\section{Figure 3}
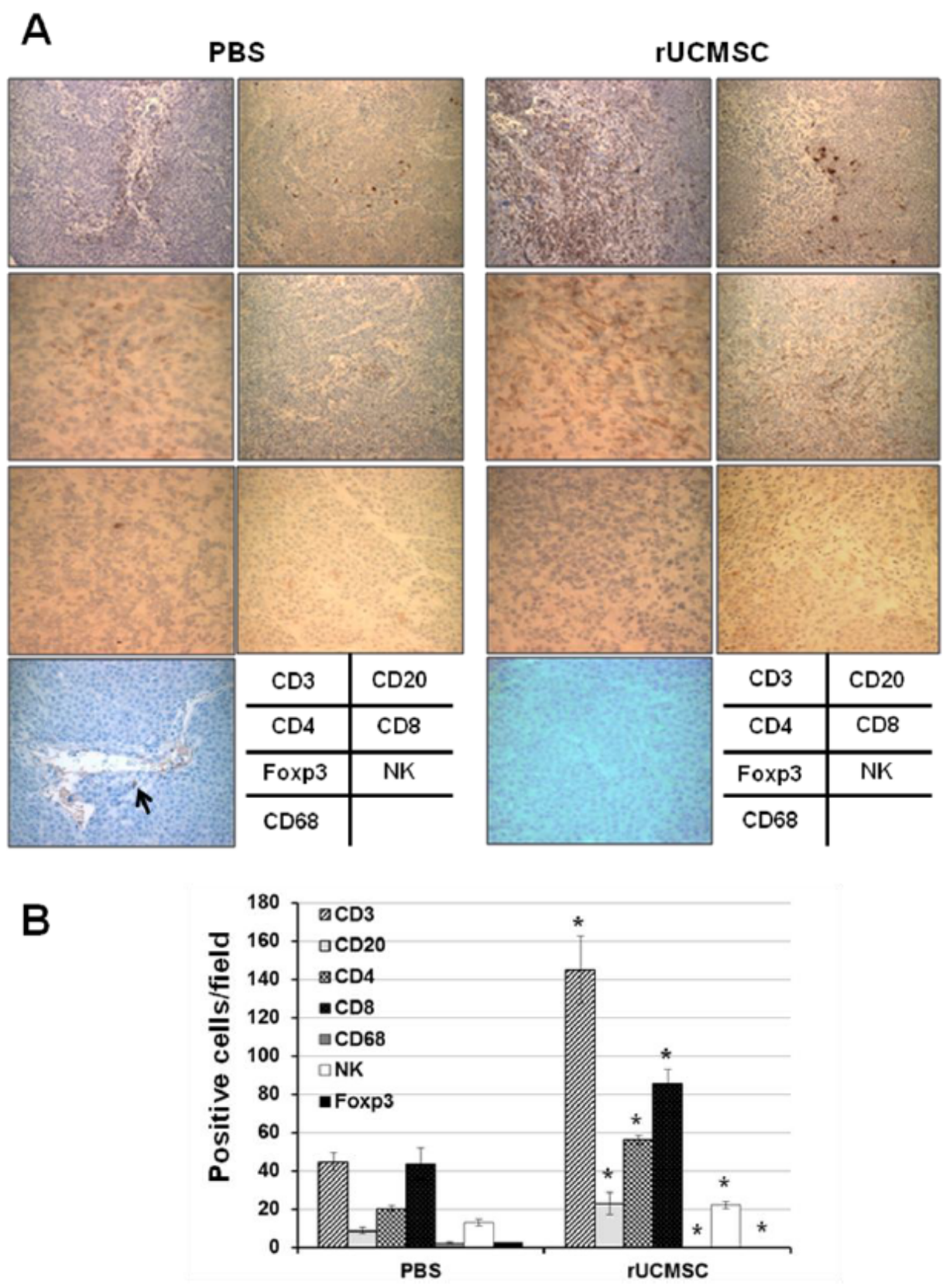

Figure 3. Detection of specific lymphocyte populations in PBS- and rUCMSC-treated tumors. rUCMSC treatment significantly stimulated infiltration of CD3, CD4, CD20,CD8+ and NK cells but attenuated Foxp3+ and CD68+ cells in Mat B III mammary tumors. Samples were prepared and subjected to immunohistochemical analysis as described in Materials and Methods. The pictures represent typical pictures of each antibody staining. The original magnification of pictures of CD3, CD20, CD8, NK marker and CD68 is x100 and of CD4 and Foxp3 is x200. The average number of each cell types (bar graphs) was determined by analyzing ten tumor areas in each treatment group and were expressed as cell number per high power (400x) field. The arrow shows a CD68+ cell. * $\mathrm{P}<0.05$ compared with PBS-treated control. 
Figure 4. Expression of MCP-1 in rat UCMSC and Mat B III cells was detected by Western blot analysis.
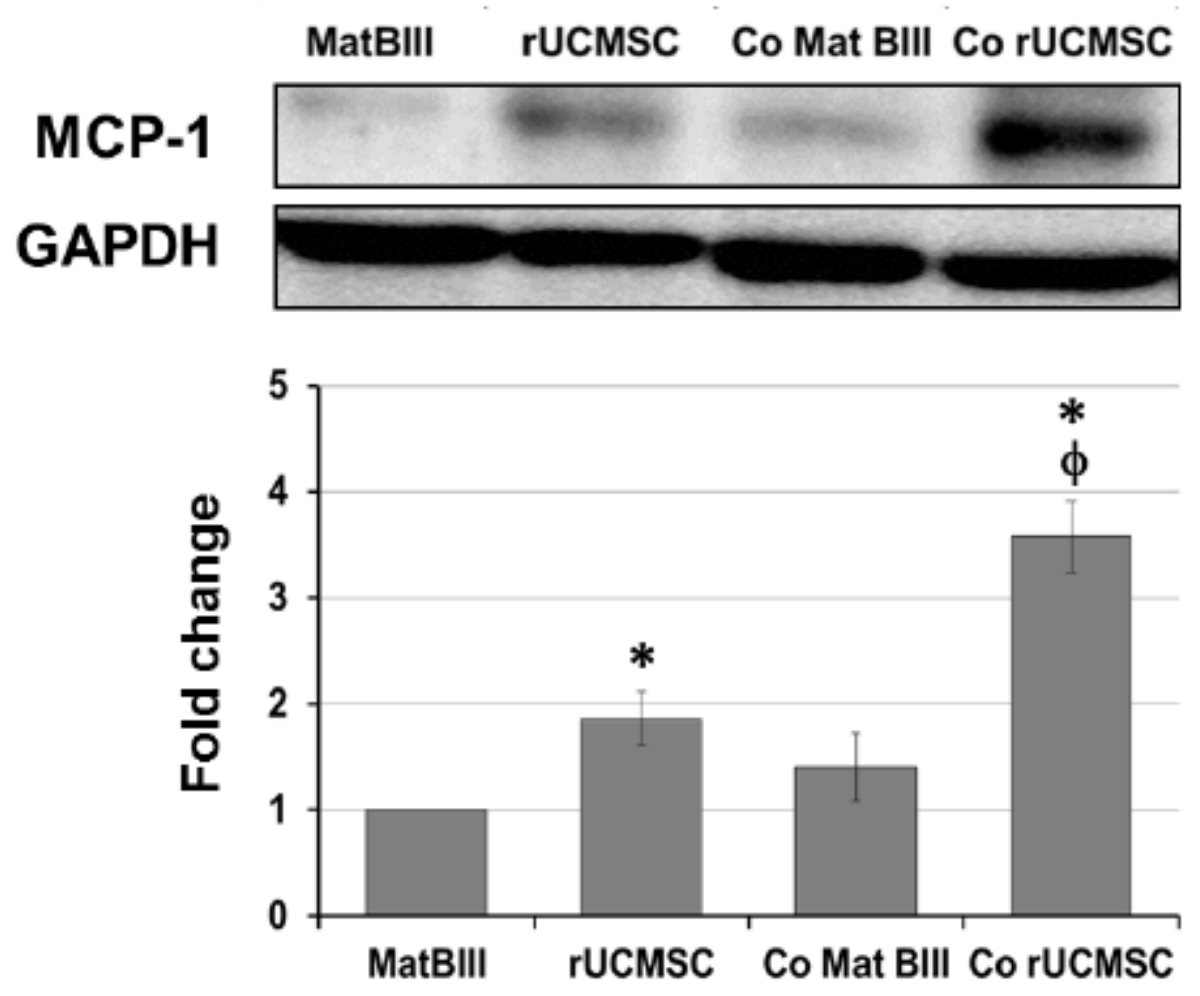

Figure 4. Indirect co-culture of a small number of rUCMSC and Mat B III significantly increased MCP-1 expression in rUCMSC. Three days after co-culture the whole cell lysate from each cell type was separately prepared and subjected to Western blot analysis. Samples were prepared as described in Materials and Methods. The average expression levels of MCP-1 were normalized by GAPDH, and are displayed in the histogram. Sample preparation and Western blot analysis were performed three times with duplicate determinations. The picture represents typical blotting results. ${ }^{*}, \mathrm{P}<0.05$ compared with Mat B III. $\phi, \mathrm{P}<0.05$ compared with rUCMSC without co-culture. 
Figure 5. Effect of anti-mouse MCP-1 antibodies on the migration of rat PBMC toward either rUCMSC or Mat B III carcinoma cells in the Boyden chamber.

A

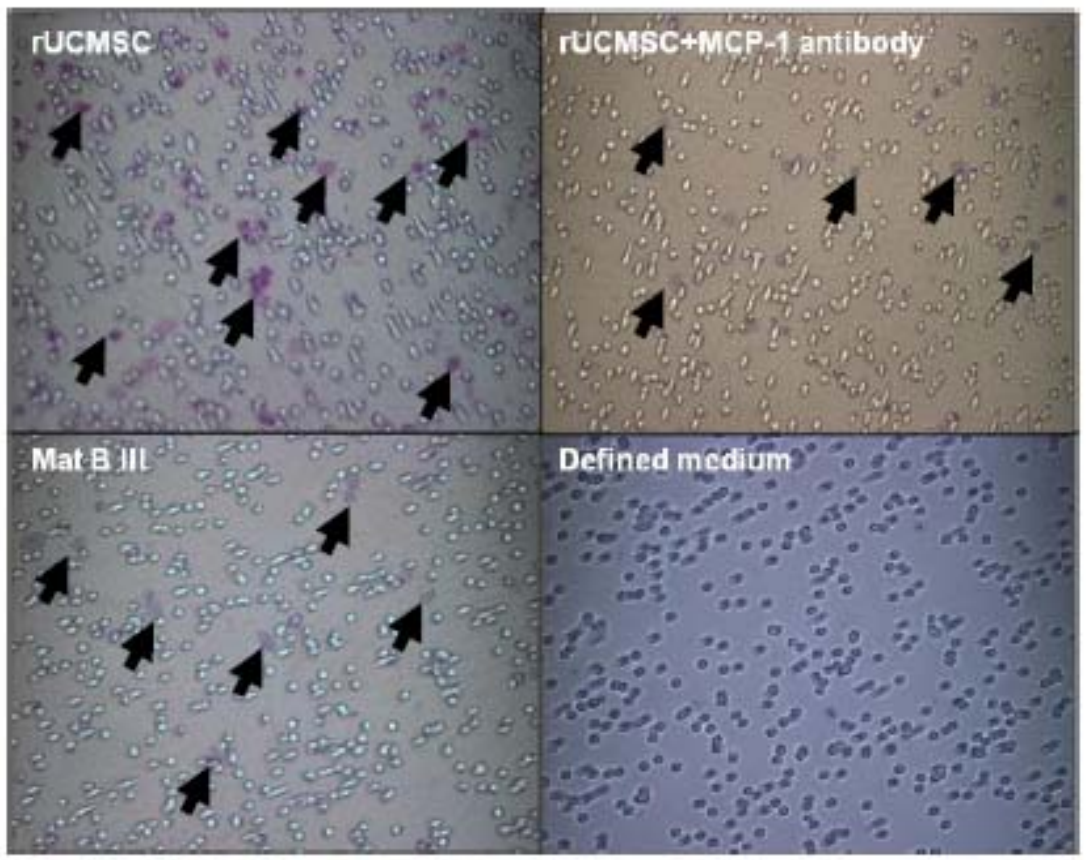

B

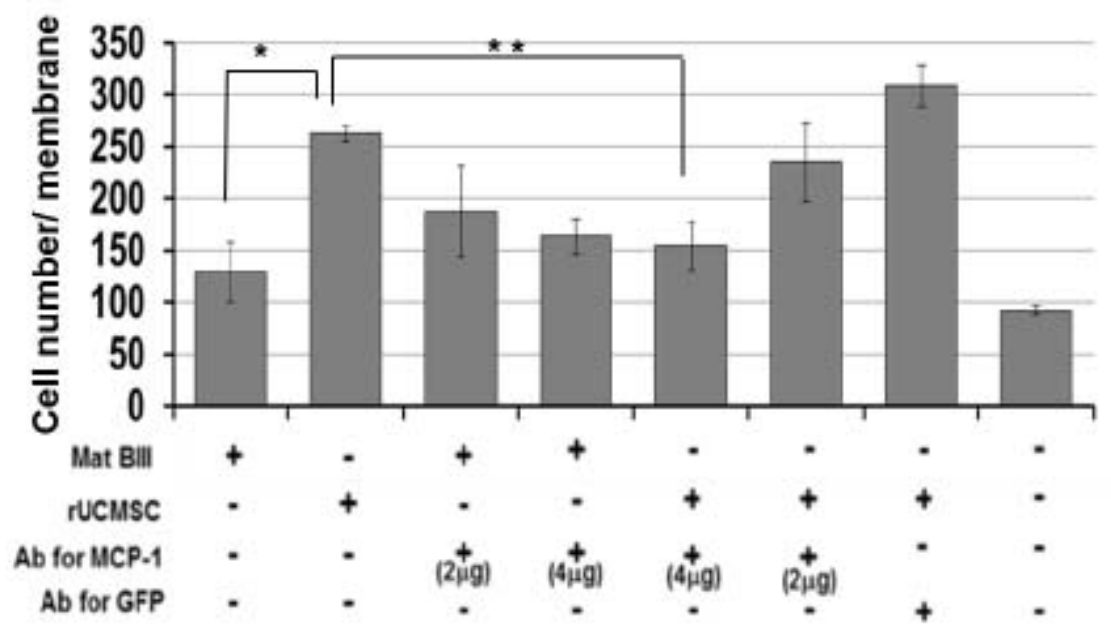

Figure 5. Addition of anti-mouse MCP-1 antibodies significantly attenuated the migration of rat PBMC (peripheral blood mononuclear cells) toward either rUCMSC or Mat B III cells in the Boyden chamber assay. Panel A shows the migrated PBMC cells (purple colored cells) on the lower surface of the membrane. Antibody against MCP-1 was added in the lower chambers as noted. Panel B shows the summarized results from the migration assay. The experiment was performed twice with triplicate determinations. Arrows show the migrated leukocytes. *, $\mathrm{P}<0.05$ compared with Mat B III. **, $\mathrm{P}<0.05$ compared with rUCMSC. 